\title{
Electromyographic studies in a case of foramen magnum meningioma ${ }^{1}$
}

\author{
J. A. LIVESON, ${ }^{2}$ J. RANSOHOFF, AND J. GOODGOLD \\ From the Electrodiagnostic Department, Institute of Rehabilitation Medicine \\ and the Department of Neurosurgery, New York University Medical Center, New York, U.S.A.
}

SUMMARY Atrophy of muscles innervated by the lower cervical cord is a well-known feature in patients with foramen magnum tumours. Electromyographic evidence of denervation in the atrophic muscles is presented. The significance and possible mechanisms are reviewed and discussed.

Tumours in the region of the foramen magnum are known to be associated with wasting of the intrinsic muscles of the hand. In several reviews (Symonds and Meadows, 1937; Martin and Kleyntjens, 1950; Love, Thelen, and Dodge, 1954; Dodge, Love, and Gottlieb, 1956; Stein, Leeds, Taveras, and Pool, 1963) the atrophy was considered an indication of pathology in the lower cervical spinal cord. The atrophy, it was assumed, implied a lower motor neurone process and therefore localized the pathology to spinal cord segments supplying the muscles of the hand.

There is no absolute specificity, however, to the phenomenon of muscle atrophy. It has, for example, been described with upper motor neurone lesions, or with simple disuse (DeJong, 1967). Because of this, the intrinsic muscle atrophy is not absolute proof that patients with foramen magnum tumours have lower cervical cord pathology.

The only evidence of pathology in the lower cervical spinal cord was reported by Stein et al. (1963). One patient had a meningioma of spinal cord segments C 1-3 and atrophy of intrinsic muscles of the hand. Histological examination of his lower cervical cord showed 'cavitation of the central region ... with preservation of the periphery.'

\footnotetext{
1 This study was supported by Grant No. 8-0162-814 from the John A. Hartford Foundation and supported in part under the designation of the New York University Medical Center as a Rehabilitation, Research and Training Center, by the Social and Rehabilitation Service, Department of Health, Education and Welfare.

2 Present address and reprint requests: Dr. Liveson, Department of Neurology, Albert Einstein College of Medicine, 1300 Morris Park Avenue, Bronx, New York 10461, U.S.A.
}

A second patient, with a meningioma at the foramen magnum and atrophy of the shoulder and hand muscles, had a normal lower cervical cord on histological examination which Stein explained by stating that 'perhaps the cellular changes were too subtle'. It is conceivable that neurophysiological rather than morphological studies could have documented the subtle changes.

Recently we had the opportunity, using neurophysiological techniques, to study a patient with a foramen magnum meningioma. Since such studies have not been described in the literature we present this brief report and a short discussion of the theoretical implications.

\section{CASE REPORT}

In March 1970 a 72 year old woman was admitted to University Hospital for evaluation of paraesthesiae of both upper extremities and slight instability of gait. Three years before admission the patient sustained severe neck flexion-extension during a car accident and noted sudden onset of tingling in the fingers of her left hand. This gradually involved the upper aspect of her left arm during the following year; urinary urgency also developed. The patient also complained of left occipital headaches which decreased while wearing a cervical collar. Several months before this first admission she had noted numbness in the right fingers and a slight unsteadiness of gait.

General physical examination was normal. No abnormalities were noted on examination of her mental status, cranial nerves and gait. Dysmetria 
was present on testing the upper but not the lower extremities. The left deltoid, biceps, wrist extensor muscles and grip were weak, with specific mention made of 'significantly less strength of intrinsic muscles of the left hand'. Sensation in the left upper extremity was decreased (joint position, graphaesthesia, point localization, and possibly light touch) with diffusely decreased vibration sensation. Deep tendon reflexes were hyperactive and symmetrical throughout. Abdominal reflexes were absent, and the left plantar response was extensor.

The results of the following tests were normal: Schilling test, complete blood count, mean corpuscular volume, mean corpuscular haemoglobin, mean corpuscular haemoglobin concentration, electrocardiogram; serum electrophoresis, serum electrolytes, serum glutamic oxalacetic and pyruvate transaminases and lactate dehydrogenase. Glucose tolerance test was mildly elevated; erythrocyte sedimentation rate was $22 \mathrm{~mm} / \mathrm{hr}$.

No abnormality was recognized on myelography of cervical through lumbar regions. Radiographs of the skull, chest, and renal tract were normal.

In the intervening 10 months until her second admission in May 1971, the patient progressively developed extreme weakness in her legs. The paraesthesiae extended to involve the right medial fore-arm and the right three medial digits.

Examination at this time revealed that her gait was broad-based, and a Romberg sign was present. There was weakness of her left upper extremity and both legs. "Atrophy of the intrinsic muscles of the left upper extremity' was noted. Sensation was diminished in her left upper extremity and in both lower extremities (touch, pin, joint position, vibration). Deep tendon reflexes were hyperactive and plantar responses were now bilaterally extensor.

MYELOGRAPHY The spinal cord was displaced to the right at the odontoid level with a filling defect at that level. A round, $4 \mathrm{~cm}$ mass in the left anterolateral position at the foramen magnum level was described as well-demarcated, intradural, and extramedullary. The cerebrospinal fluid protein was $33 \mathrm{mg} / 100 \mathrm{ml}$.

On 1 June 1971 a tumour was removed through suboccipital craniectomy, and laminectomy of $\mathrm{Cl}$ and $\mathrm{C} 2$ vertebrae. The cervico-medullary junction appeared displaced to the right and the cerebellum was elevated. With the aid of the operating microscope, a well-encapsulated tumour was removed from the posterior fossa under the medulla with its lower pole at the $\mathrm{Cl}$ vertebral level. Several small vessels and nerve roots were stretched over the lower end. After the tumour was removed (one specimen measuring larger than 3 by $2 \mathrm{~cm}$ ) the left vertebral artery was seen to be displaced laterally and elevated. $\frac{\text { O }}{Z}$ A small vessel stretched from the vertebral artery to the cord.

On neuropathological examination, interlacing $\stackrel{0}{\subseteq}$ bundles of fusiform cells and multiple whorls with focal calcification were seen. The diagnosis was 0 meningioma.

Post-operatively the patient was confused for $\frac{O}{\overline{0}}$. several days and she had right upper extremity dysmetria and spastic left-sided weakness. Steroids were given. During the next 10 days her confusion $\underset{\overrightarrow{\mid}}{\vec{P}}$ cleared, and the dysmetria and weakness decreased. By discharge two weeks post-operatively she had $\frac{}{\sigma}$ mild left hemiparesis, no dysmetria, some return of $\frac{\bar{m}}{\bar{D}}$ sensation (joint position), and flexor plantar re- $\mathbb{\mathbb { Q }}$ sponses. She has continued to improve over the six on months since discharge.

ELECTRODIAGNOSTIC TESTS Electromyography was $\overrightarrow{-}$ performed using Teca concentric electrodes with $\vec{\sigma}$ Grass P511R and Tektronix 3A3 amplifiers. Motor nerve conduction was studied using supramaximal square wave electrical stimuli of $0 \cdot 1-0 \cdot 3 \mathrm{msec}$ dura- $\omega$ tion, and $1 / \mathrm{sec}$ frequency delivered by a Grass $S=8$ stimulator coupled through a General Radio isoles is tion transformer. Surface electrodes were placent $\triangle$ over the abductor pollicis brevis muscle for mediag nerve studies, and the abductor digiti quinti muscfefor ulnar nerve studies. Latencies of sensory nerke? action potentials were determined using antidromico stimulation at the wrist and monitoring the response क using surface electrodes on the second digit (mediat) $\vec{\omega}$ or fifth digit (ulnar) (Campbell, 1962; Goodgold ant Eberstein, 1972).

In March 1970 electromyography and nerve conduction studies of both upper extremities were normal.

In May 1971 the electromyographic findings were $\frac{0}{\varnothing}$ different. On the left side fibrillation potentials and $\stackrel{\mathbb{Q}}{2}$ positive sharp waves were present in the brachio- $\overrightarrow{\overrightarrow{0}}$ radialis, flexor carpi radialis and ulnaris, extensor 3 digitorum communis, first dorsal interosseous, and abductor pollicis brevis muscles. On the right, the? same abnormal spontaneous discharges were noted in the deltoid and first dorsal interosseous. Bizarre? high-frequency discharges were noted in the left upper trapezius muscle. No abnormality was found in other muscles of the upper extremities, nor in any muscles $\delta$ of the lower extremities. Conduction studies were normal, including ulnar motor nerve conduction 을 bilaterally, and sensory nerve action potential deter- $D$ minations of the left median and ulnar nerves.

\section{DISCUSSION}

The patient's clinical course and symptoms 0 included (1) occipital headaches, (2) progression N 
of numbness and weakness which started in one upper extremity and eventually involved all the extremities, and (3) atrophy of the intrinsic muscles of the hands. These features have been described previously as typical of foramen magnum tumours by several authors (Elsberg and Strauss, 1929; Symonds and Meadows, 1937; Abbott, 1950; Martin and Kleyntjens, 1950; Love et al., 1954; Dodge et al., 1956; Hensen and Parsons, 1967; Epstein, Epstein, and Carras, 1970).

Electrodiagnostic studies were normal at the time of the first admission and clinical examination showed only slight weakness of the intrinsic muscles of the left hand. Atrophy of the intrinsic muscles of the hands was not described until 14 months later and was associated with findings of fibrillation potentials and positive sharp waves on electromyographic examination. Similar abnormal electrical findings were present in other muscles supplied by C5-T1 roots. (The abnormality in the left trapezius muscle was probably due to involvement of the left accessory nerve, although injury to the C3-4 roots is an alternative explanation).

With regard to the electromyographic findings, it is interesting to note that spontaneous abnormal activity (fibrillation potentials, positive sharp waves) have been observed in the limb muscles of patients with upper motor neurone lesions. Rosen, Lerner, and Rosenthal (1969) reported these findings in six of seven patients with traumatic lesions between $\mathrm{C} 5$ and $\mathrm{T} 10$ spinal cord segments. Nyboer and Johnson (1971) found similar abnormalities in eight of 10 patients with trauma to the cervical cord. Similar spontaneous abnormal activity has been reported in some series of hemiplegic patients due to cerebral lesions (Goldkamp, 1967; Bhala, 1969; Notermans and Blokzijl, 1969; Spielholz, Sell, Goodgold, Rush, and Greens, 1971; but see Alpert, Idarraga, Orbegozo, and Rosenthal, 1971). It has been postulated that the activity reflects a trophic disturbance of the upper motor neurone on the anterior horn cell.

In the present case, although the Babinski signs imply bilateral upper motor neurone dysfunction of the lower extremities, it is significant that the lower extremities did not reveal any abnormal electrical findings. This argues against an upper motor neurone lesion as an explanation for the electrical abnormalities restricted to the hands. It is also unlikely that the atrophy was caused by disuse, since in no cases of disuse atrophy have fibrillation potentials and positive sharp waves been reported. It is our conclusion, therefore, that the neurophysiological evidence favours anterior horn cell dysfunction of the lower cervical cord, rather than upper motor neurone dysfunction or disuse atrophy.

Previous authors (Symonds and Meadows, 1937; Martin and Kleyntjens, 1950; Love et al., 1954; Stookey, 1954; Dodge et al., 1956; Stein et al., 1963) have postulated a vascular mechanism as the basis for the lower cervical cord abnormalities. This theory was supported by Bolton (1939) who injected the vertebral arteries of four cadavers and traced the blood flow to the spinal cord. He noted narrowing of the anterior spinal artery above its junctions with large radicular arteries, haemodynamically facilitating downward flow of blood at these junctions. In the areas above the narrowing, the anterior spinal artery effectively functions as an end artery, making these zones most vulnerable to vascular deficit ('watershed' areas). The most marked of such zones in the distribution of the anterior spinal artery is at the level of the upper thoracic segments. The vascular supply of the lower cervical cord has been described as primarily dependent on the anterior spinal artery which is formed by the junction of two branches of the vertebral arteries in the posterior fossa (Schneider and Schemm, 1961 ; Turnbull, Brieg, and Hassler, 1966). This could provide an explanation for the remote effects of a posterior fossa foramen magnum lesion on the lower cervical cord.

Doubts have been raised about the postulated vascular mechanism. More recent studies have shown that the major blood flow to the anterior spinal cord may be via the large radicular arteries in the neck, with a relatively minor contribution from the vertebral arteries. Gillilan $(1958,1970)$, discounts any significant contribution from the vertebral branches. Fried, Doppman, and DiChiro (1970) demonstrated virtually no supply from the vertebral arteries on brachial angiography of five monkeys.

In spite of these objections it is possible that there are variations among individuals so that some have a slightly greater contribution from the vertebral supply. Turnbull et al. (1966) 
studied cord blood supplies and demonstrated great variability. If the major proportion of blood to the cervical-thoracic region is not primarily from the lower radicular arteries in the neck, vertebral flow would have a crucial effect on the 'watershed' region. This variation pertained in $10 \%$ of Turnbull's necropsy series who lacked radicular arteries in the cervical region. The specific haemodynamic situation in each individual case would determine the contribution, if any, of the vertebral artery branches.

Data on the vascular supply in patients with foramen magnum tumours is lacking. In the second case that came to necropsy, Stein et al. (1963) noted that 'the vertebral artery did not appear to be compressed. Although the anterior spinal artery ran through the zone of compression, it was not occluded.' It may be argued that their patient had haemodynamic changes rather than actual obliterative changes. If that is the case arteriographic studies might demonstrate this. Bull (1969) reported that a large posterior fossa aneurysm can simulate a mass near the foramen magnum. Angiography in these patients is thus indicated for diagnostic reasons, in addition to casting light on the puzzling neurological findings.

\section{REFERENCES}

Abbott, K. H. (1950). Foramen magnum and high cervical cord lesions simulating degenerative disease of the nervous system. Ohio State Medical Journal, 46, 645-651.

Alpert, S., Idarraga, S., Orbegozo, J., and Rosenthal, A. M. (1971). Absence of electromyographic evidence of lower motor neuron involvement in hemiplegic patients. Archives of Physical Medicine and Rehabilitation, 52, 179-181.

Bhala, R. P. (1969). Electromyographic evidence of lower motor neuron involvement in hemiplegia. Archives of Physical Medicine and Rehabilitation, 50, 632-637.

Bolton, B. (1939). The blood supply of the human spinal cord. Journal of Neurology, Neurosurgery, and Psychiatry, 2, 137-148.

Bull, J. (1969). Massive aneurysms at the base of the brain. Brain, 92, 535-570.

Campbell, E. D. R. (1962). The carpal tunnel syndrome: investigation and assessment of treatment. Proceedings of the Royal Society of Medicine, 55, 401-405.

DeJong, R. N. (1967). The Neurologic Examination, 3rd edn., pp. 512-521. Harper: New York.

Dodge, H. W., Jr., Love, J. G., and Gottlieb, C. M. (1956).
Benign tumors at the foramen magnum: surgical considerations. Journal of Neurosurgery, 13, 603-617.

Elsberg, C. A., and Strauss, I. (1929). Tumors of the spinal cord which project into the posterior cranial fossa. Archives of Neurology and Psychiatry, 21, 261-273.

Epstein, B. S., Epstein, J. A., and Carras, R. (1970). Extension of posterior fossa tumors, particularly intraventricular fourth ventricle tumors, into the upper cervical spinal canal. American Journal of Roentgenology, 110, 31-38.

Fried, L. C., Doppman, J. L., and Di Chiro, G. (1970). Direction of blood flow in the primate cervical spinal cord. Journal of Neurosurgery, 33, 325-330.

Gillilan, L. A. (1971). Arterial and venous anatomy of the spinal cord. In Cerebrovascular Diseases, 7th Conference, 1970, pp. 3-9. Edited by J. F. Toole, J. Moossy, and R. Janeway. Grune and Stratton: New York.

Gillilan, L. A. (1958). The arterial blood supply of the human spinal cord. Journal of Comparative Neurology, 110, 75-103.

Goldkamp, O. (1967). Electromyography and nerve conduction studies in 116 patients with hemiplegia. Archives of Physical Medicine and Rehabilitation, 48, 59-63.

Goodgold, J., and Eberstein, A. (1972). Electrodiagnosis of Neuromuscular Diseases, pp. 41-59, 80-115. Williams and Wilkins: Baltimore.

Hensen, R. A., and Parsons, M. (1967). Ischaemic lesions of the spinal cord: an illustrated review. Quarterly Journal of Medicine, 36, 205-222.

Love, J. G., Thelen, E. P., and Dodge, H. W., Jr. (1954). Symposium: tumors of the foramen magnum. Journal of the International College of Surgeons, 22, 1-17.

Martin, P., and Kleyntjens, F. (1950). Tumeurs sous-durales du trou occipital. Revue Neurologique, 82, 313-334.

Notermans, S. L. H., and Blokzijl, E. J. (1969). Electro-@ myography in patients with lesions of the central motor neuron and the so-called parietal muscular atrophy. Psychiatrica, Neurologia Neurochirurgia, 72, 557-567.

Nyboer, V. J., and Johnson, H. E. (1971). Electromyographic $\bigcirc$ findings in lower extremities of patients with traumatic quadriplegia. Archives of Physical Medicine and Rehabilitation, 52, 256-259.

Rosen, J. S., Lerner, I. M., and Rosenthal, A. M. (1969). Electromyography in spinal cord injury. Archives of Physical Medicine, 50, 271-273.

Schneider, R. C., and Schemm, G. W. (1961). Vertebral artery insufficiency in acute and chronic spinal trauma. Journal of Neurosurgery, 18, 348-360.

Spielholz, N. I., Sell, G. H., Goodgold, J., Rush, H., and Greens, S. K. (1971). Electrophysiologic studies in patients with spinal cord lesions. (Abstract.) Archives of Physical Medicine and Rehabilitation, 52, 588-589.

Stein, B. M., Leeds, N. E., Taveras, J. M., and Pool, J. L. (1963). Meningiomas of the foramen magnum. Journal of Neurosurgery, 20, 740-751.

Stookey, B. (1954). Symposium: tumors of the foramen magnum. Journal of the International College of Surgeons, 22, 14-15. Discussion in Love, J. G., Thelen, E. P., and Dodge, H. W., Jr. (1954).

Symonds, C. P., and Meadows, S. P. (1937). Compression of the spinal cord in the neighbourhood of the foramen magnum. Brain, 60, 52-84.

Turnbull, I. M., Brieg, A., and Hassler, O. (1966). Blood supply of cervical spinal cord in man. Journal of Neurosurgery, 24, 951-965. 